\title{
Successful Secondary Endovascular Intervention in Pediatric Patients with Venous Thromboembolic Events
}

\author{
Hannah Glonnegger ${ }^{1} \quad$ Barbara Zieger ${ }^{1} \quad$ Jochen Grohmann ${ }^{2,3} \quad$ Gabriele Freund $^{4} \quad$ Thomas Zeller $^{4}$ \\ Markus Uhl ${ }^{5}$ Brigitte Stiller ${ }^{2}$
}

${ }^{1}$ Division of Pediatric Hematology and Oncology, Department of Pediatrics and Adolescent Medicine, Faculty of Medicine, Medical Center - University of Freiburg, Germany

${ }^{2}$ Department of Congenital Heart Defects and Pediatric Cardiology, Faculty of Medicine, University Heart Center Freiburg - Bad Krozingen, University of Freiburg, Freiburg im Breisgau, Germany

3 Department of Congenital Heart Disease/Pediatric Cardiology, Heart and Diabetes Center NRW, Ruhr-University Bochum, Bad Oeynhausen, Germany

${ }^{4}$ Department of Angiology, Faculty of Medicine, University Heart Center Freiburg - Bad Krozingen, University of Freiburg, Freiburg im Breisgau, Germany

${ }^{5}$ Division of Pediatric Radiology, Department of Radiology, Faculty of Medicine, University of Freiburg, Freiburg im Breisgau, Germany

Hamostaseologie
Address for correspondence Barbara Zieger, MD, Department for Pediatric Hematology and Oncology, University Medical Center Freiburg, Mathildenstraße 1, 79106 Freiburg, Germany (e-mail: barbara.zieger@uniklinik-freiburg.de).

\section{Abstract}

Keywords

- endovascular intervention

- children

- venous thromboembolic event
Background In the past, pediatric patients with venous thromboembolic events (VTE) were treated with low-molecular-weight heparin (LMWH) which was successful in around $70 \%$ of the cases. However, anticoagulation alone might not restore patency in all patients, and advanced therapeutic options to prevent postthrombotic syndrome are needed. During recent years, endovascular interventions have become a treatment option for pediatric patients with persistent thrombotic occlusion, not only in life- or limb-threatening VTE.

Methods We evaluated 12 consecutive patients (11-17 years) with newly diagnosed VTE being treated at our department during the last 4 years (2017-2020). In case follow-up examination showed persistent venoocclusion under anticoagulation, patients received secondary interventional therapy like recanalization, percutaneous transluminal angioplasty with or without catheter-directed thrombolysis, and stenting. Patients with no clinical signs of venoocclusion or regredient thrombosis in imaging examination received anticoagulation alone.

Results Six of $12(50 \%)$ patients underwent catheter intervention. Median time from diagnosis to intervention was 4 months ( $0-12$ months). Reintervention was necessary in one $(8 \%)$ case and complete recanalization failed in one $(8 \%)$ case. There were no major bleeding events or other major postinterventional complications, no acute or late local recurrence, and all patients reported clinical improvement after the procedure. received

July 18,2021

accepted after revision

November 18, 2021 (c) 2022. Thieme. All rights reserved. Georg Thieme Verlag KG,

Rüdigerstraße 14,

70469 Stuttgart, Germany
DOI https://doi.org/

10.1055/a-1701-2098

ISSN 0720-9355. 
Conclusion If endovascular intervention is used in teenage patients with persistent symptomatic VTE, reduction of postthrombotic symptoms is possible, even if intervention is performed secondary to failure of anticoagulation. Multidisciplinary treatment decisions can be based on the clinical course and follow-up imaging.

\section{Introduction}

Venous thromboembolism (VTE) affects 0.07 to $0.14 / 10.000$ of all children. ${ }^{1,2}$ However, there seems to be a substantial increase in cases since the 1990 s, mainly due to a higher rate of malignant diseases being treatable and a higher rate of pediatric patients having central venous lines. ${ }^{3-5}$

VTE is a multivariate condition. In children, Peter and coworkers reported that around $90 \%$ of cases are associated with prothrombotic risk factors. ${ }^{2}$ Risk factors comprise hereditary deficiencies or mutations in coagulation factors, autoimmune diseases, malignant conditions, and anatomic risk factors, as well as transient risk factors like immobilization, infection, or intake of certain medications. ${ }^{6,7}$

Differences in underlying risk factors, associated chronic diseases, and natural course of thrombotic closure impose a specific pediatric approach to therapeutic strategies. For example, central venous lines are a common risk factor for thrombosis in children, whereas VTE recurrence is less common in children compared with adults. ${ }^{8}$ Additionally, the occurrence of concurrent problems like postthrombotic syndrome and chronic thrombotic closure demand further examination of alternative treatment options besides therapeutic anticoagulation, like it has been already established in adult patients. Specific interest should be given to the optimal treatment in children, as the possibility to enhance quality-adjusted life-years increases significantly.

Current initial treatment of choice in pediatric patients is the use of anticoagulants. Dosing of heparin in children was established by Massicotte et al. ${ }^{9}$ Treatment time is recommended for 6 weeks to 3 months in children with identified risk factors and 6 to 12 months in children with idiopathic VTE (CHEST guidelines). ${ }^{10}$ In case of specific hereditary predisposition, long-term or even life-long anticoagulation may be necessary. ${ }^{11}$

Thrombolysis has been used as a treatment option for children with life- or limb-threatening VTE. ${ }^{11}$ Since postthrombotic syndrome occurs in up to $70 \%$ of children with total venous occlusion, interventional treatment options should be considered even in non-life- or limb-threatening deep vein thrombosis (DVT). ${ }^{12,13}$ Thus, during recent years different strategies of thrombolysis have been successfully used in pediatric patients. ${ }^{14}$ Current CHEST recommendations suggest use of thrombolysis in acute DVT for young patients with a prospected long lifespan and low risk of bleeding but only in cases where intervention can be safely performed. All other cases should receive anticoagulant therapy. ${ }^{10,15}$ Regarding time point of endovascular intervention, thrombolysis has been recommended within 60 days post diagnosis. However, recent findings in adults suggest that successful restoration of venous flow can be achieved even later than 60 days. ${ }^{16,17}$

Different strategies of endovascular intervention can be applied to restore venous flow. First, recanalization must be performed using various devices. Percutaneous transluminal angioplasty (PTA) uses a catheter that is placed through the vessel and once the catheter is in place, a balloon is inflated to further reopen the vessel. Catheter-directed thrombolysis is a pharmacological strategy administering mostly recombinant-tissue plasminogen activator ( $\mathrm{r}$-tPA) continuously into the affected vein distal of the thrombus. Also systemic lysis can be applied; however, bleeding risk increases relevantly up to $15 \%$ of cases. ${ }^{18}$ Concurrent anticoagulation is necessary in both cases. Other techniques include percutaneous mechanical thrombolysis where the thrombus is removed mechanically and pharmacomechanical catheter-directed thrombolysis where after mechanical removal thrombolysis is administered locally. Percutaneous mechanical thrombolysis and pharmacomechanical catheter-directed thrombolysis seem to be the most commonly used technique in children, ${ }^{19}$ but they also increase the risk of vessel injury.

As data on interventional strategies in children with VTE are still scarce, we aimed to investigate all cases treated with interventional strategies for VTE in our center regarding safety and efficacy. In addition, we hypothesized that even late intervention ( $>60$ days post diagnosis) may be successful and safe. Furthermore, we also describe patients of our cohort who did not receive endovascular intervention and investigated their outcome.

\section{Methods}

\section{Patients}

Retrospective data collection of 12 consecutive pediatric cases with VTE was performed by a clinician regularly examining outpatients at our department of hemostaseology.

Study inclusion criteria were as follows ${ }^{1}$ : diagnosis of VTE; objectively confirmed by compression duplex ultrasound, magnet resonance angiography (MRA), or computed tomography (CT) angiography ${ }^{2}$; time of diagnosis from February 2017 to October $2020^{3}$; age less than 18 years at diagnosis $^{4}$; follow-up examination via compression duplex ultrasound or MRA.

\section{Indication for Intervention}

Initial treatment of all patients was unfractionated heparin (for $\sim 24$ hours) followed by therapeutic dosage of lowmolecular-weight heparin (LMWH). Regular follow-up examinations were performed on the ward (daily) and in 
the outpatient department (at least every 4 weeks). Indication for a secondary intervention included (a) continued clinical signs of veno-occlusion (swelling, pain, discoloration, massive collateralization) and (b) MRA and compression duplex ultrasound with persistent complete occlusion. If residual flow was seen in imaging, a short follow-up examination was performed to evaluate reperfusion progress. A consensus statement was achieved involving the section of pediatric hemostaseology, the center for vascular diseases, and the pediatric cardiac interventionalists.

The intervention was performed by the pediatric cardiac interventionalists or members of the center for vascular diseases using PTA and/or catheter-directed thrombolysis. Stents were placed in case of residual thrombus or stenosis.

\section{Data Collection}

The retrospective systematic collection of clinically derived data included patient demographics, time of symptoms onset, thrombus location, clinical findings at presentation, risk factors for VTE, the presence of May-Thurner anomaly, interventions performed, postinterventional outcome, and follow-up results.

\section{Risk Factors for Thrombophilia}

- Presence of factor V-Leiden mutation (homo-/ heterozygous).

- Presence of factor II mutation (homo-/heterozygous).

- Protein C, protein S, antithrombin deficiency. Genetic testing was performed to differentiate between hereditary and acquired reduction.

- Hypodysfibrinogenemia.

- Antiphospholipid antibodies and antinuclear antibodies, respectively, which persisted more than 3 months from onset of symptoms.

\section{Radiologic Imaging}

Most patients received MRA and duplex ultrasound as initial investigation and as follow-up, whereas some patients received only duplex ultrasound as follow-up due to optimal visualization.

In case of persistent clinical symptoms after intervention, a reintervention was scheduled that included fluoroscopy to establish the extent of chronic thrombus burden or residual stenosis.

\section{Statistical Methods}

Descriptive statistics were used to characterize continuous data and frequencies of categorical data.

\section{Results}

A total of 12 patients met the inclusion criteria. Six of 12 (50\%) patients underwent endovascular intervention, whereas 6 of 12 (50\%) patients were treated with anticoagulation alone.

Study population demographics, baseline patient characteristics, thrombus characteristics, and VTE risk factors are shown in -Table 1.
Median age was 14 years (11-17.1 years). Ten of 12 ( $83 \%)$ patients suffered from pelvic vein thrombosis, and 2 of 12 (7\%) patients had thrombotic closure of the inferior vena cava (IVC). May-Thurner anomaly was diagnosed in 2 of 12 (17\%) of cases.

Interventional therapy was performed in 6 patients because of (a) clinical signs of claudication (3/12; 25\%), (b) continuous extensive thrombosis $(2 / 12 ; 17 \%)$, or (c) impaired prehepatic flow $(1 / 12 ; 8 \%)$. If the patient did not show any clinical signs of claudication and patent collateralization was already shown or if thrombus resolved under therapeutic anticoagulation, intervention was not performed.

No IVC filters were used in this cohort.

Time from diagnosis to intervention was 4 (1-12 months) months. Five of 12 (42\%) patients received stenting during intervention. Applied techniques included PTA only in 5 of 12 (42\%) cases and PTA plus catheter-directed thrombolysis in 2 of 12 (17\%) cases. Techniques, outcomes, and anticoagulant regime are shown in - Table 2. - Fig. 1 shows patient number 10 with an IVC thrombosis and successful recanalization.

\section{Safety and Efficacy}

There were no periprocedural major bleeding complications during or after intervention. One patient suffered from minor puncture site bleeding post intervention. No pulmonary embolism occurred.

Full technical success was achieved in five if six (83\%) cases within the first procedure. In one case, recanalization was only partially possible (until lumbosacral transition zone) and in one case recanalization failed at the first intervention and needed to be repeated in a second procedure ( $\sim$ Fig. 2). In one case, redilatation was necessary after 7 months due to in-stent stenosis. Otherwise, there was no acute local recurrence and no late recurrence.

\section{Outcome}

All patients reported clinical improvement after the procedure and MRA or ultrasound follow-up exams showed sustained flow at intervention site. Four of six (67\%) patients did not show any signs of postinterventional residual thrombosis or in-stent thrombosis, and three (50\%) demonstrated a smaller caliber of the affected vessel or minimal residual thrombosis.

In patients not receiving intervention, follow-up showed either partial or complete reperfusion or patent (sufficient) collateralization and no signs of postthrombotic syndrome.

Clinical follow-up showed mild differences in lower limb circumference in 2 of 12 (17\%) patients (one in intervention and one in nonintervention group) and one patient had mild abdominal caput medusa in nonintervention group. Nine of the 12 (75\%) patients do not have any clinical symptoms of DVT or postthrombotic syndrome. Mean follow-up time was 26 months.

\section{Risk Factors}

With regard to the question whether patients with thrombophilic risk factors are more likely to suffer from symptomatic closure and therefore undergo intervention after initial 
Endovascular Intervention in Pediatric Patients Glonnegger et al.

\begin{tabular}{|c|c|c|c|c|c|c|c|c|c|c|c|c|}
\hline 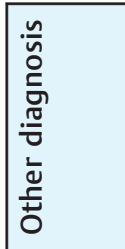 & & & & & & & & & & 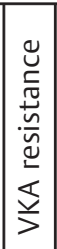 & $\frac{n}{\frac{1}{\alpha}}$ & 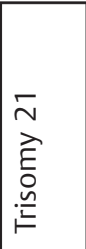 \\
\hline 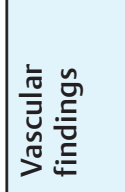 & 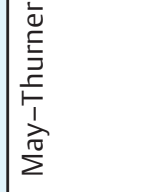 & & 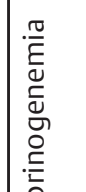 & & & & & 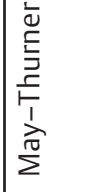 & & & & \\
\hline 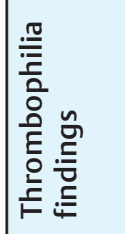 & 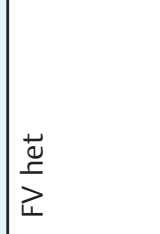 & 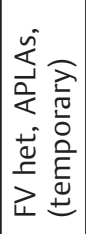 & 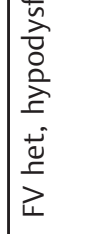 & 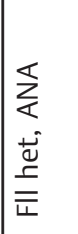 & 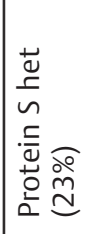 & 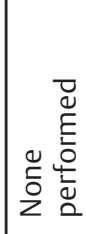 & 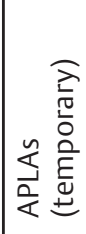 & 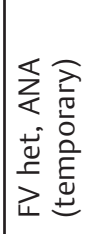 & 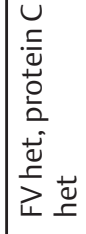 & 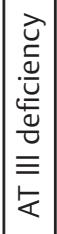 & 妾 & 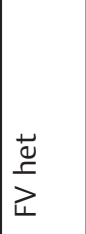 \\
\hline 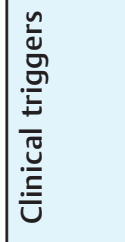 & 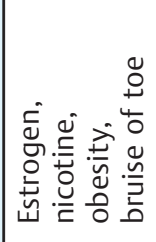 & $\frac{\varrho}{\frac{\rho}{4}}$ & 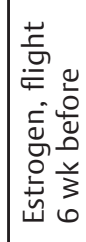 & $\begin{array}{l}0 \\
\tilde{\check{0}} \\
z\end{array}$ & 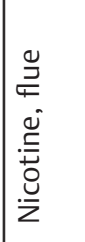 & 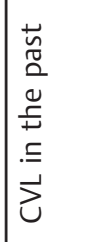 & $\begin{array}{l}0 \\
\tilde{\check{0}} \\
z\end{array}$ & 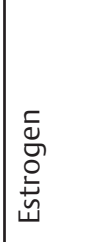 & 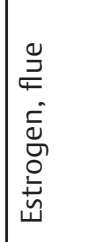 & $\mid \begin{array}{l}\overrightarrow{\vec{v}} \\
\overrightarrow{\tilde{u}} \\
\overrightarrow{0} \\
0\end{array}$ & 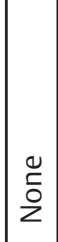 & 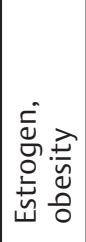 \\
\hline 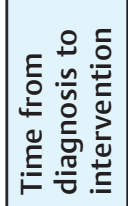 & \begin{tabular}{|l}
$\circ$ \\
$\Xi$ \\
$\sigma$
\end{tabular} & \begin{tabular}{|l}
$\stackrel{े}{E}$ \\
$\dot{\sigma}$
\end{tabular} & $\begin{array}{l}\stackrel{O}{E} \\
\stackrel{\sim}{\simeq}\end{array}$ & $\begin{array}{l}\stackrel{\circ}{E} \\
-\end{array}$ & $\begin{array}{l}\stackrel{O}{E} \\
\stackrel{\text { V }}{N}\end{array}$ & $\begin{array}{l}\stackrel{\circ}{E} \\
\stackrel{\nabla}{\sigma}\end{array}$ & & & & & & \\
\hline 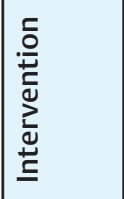 & $\stackrel{\check{\nu}}{\nu}$ & $\stackrel{\check{\nu}}{\nu}$ & $\stackrel{\check{\nu}}{\check{\nu}}$ & $\stackrel{\check{\nu}}{\nu}$ & $\stackrel{\tilde{u}}{\varnothing}$ & $\stackrel{\check{\nu}}{\nu}$ & $\mid \begin{array}{l}0 \\
\check{0} \\
2\end{array}$ & $\begin{array}{l}0 \\
\check{0} \\
\check{z}\end{array}$ & 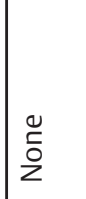 & 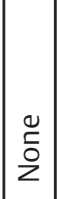 & $\begin{array}{l}0 \\
\check{0} \\
2 \\
z\end{array}$ & 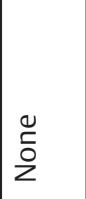 \\
\hline 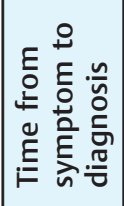 & $\begin{array}{l}\nabla \\
m\end{array}$ & $\begin{array}{l}\nabla \\
m\end{array}$ & $\begin{array}{l}\sigma \\
\mathrm{m}\end{array}$ & - & r & 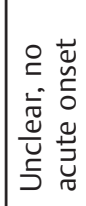 & 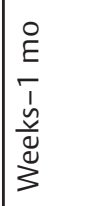 & 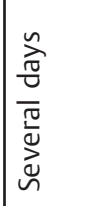 & 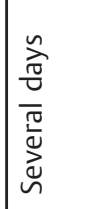 & 0 & $\begin{array}{l}\frac{y}{3} \\
\sim\end{array}$ & $\frac{\text { L }}{3}$ \\
\hline 㟔 & & & & & & & 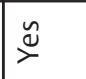 & $\stackrel{\tilde{u}}{\nu}$ & & & $\stackrel{\tilde{u}}{\succ}$ & \\
\hline 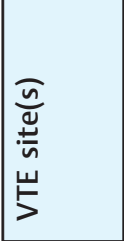 & 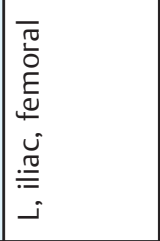 & 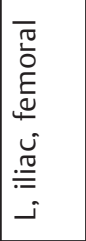 & 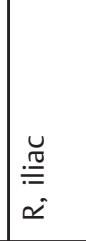 & 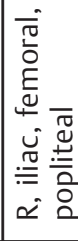 & 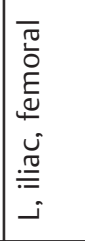 & 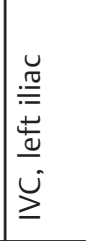 & 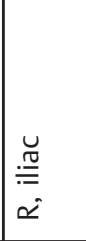 & 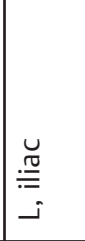 & 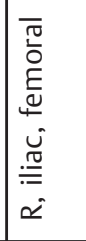 & 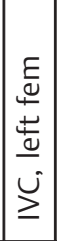 & 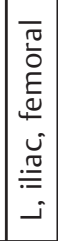 & 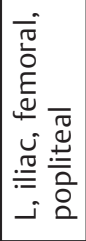 \\
\hline 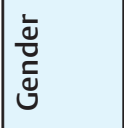 & 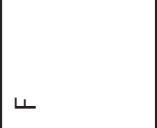 & $\sqcup$ & ப & ப & $\Sigma$ & $\Sigma$ & ப & ப & ப & $\Sigma$ & $\Sigma$ & 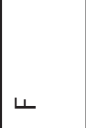 \\
\hline 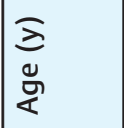 & $\stackrel{0}{\circ}$ & $\stackrel{m}{r}$ & $\dddot{0}$ & $\stackrel{\nabla}{ \pm}$ & $\approx$ & $\cong$ & $\stackrel{m}{ }$ & $\approx$ & 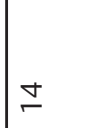 & $=$ & $\because$ & $\nexists$ \\
\hline$\dot{y}$ & - & 0 & $m$ & 6 & $\infty$ & $\circ$ & $\nabla$ & Ln & $\wedge$ & a & $\mp$ & $\simeq$ \\
\hline
\end{tabular}




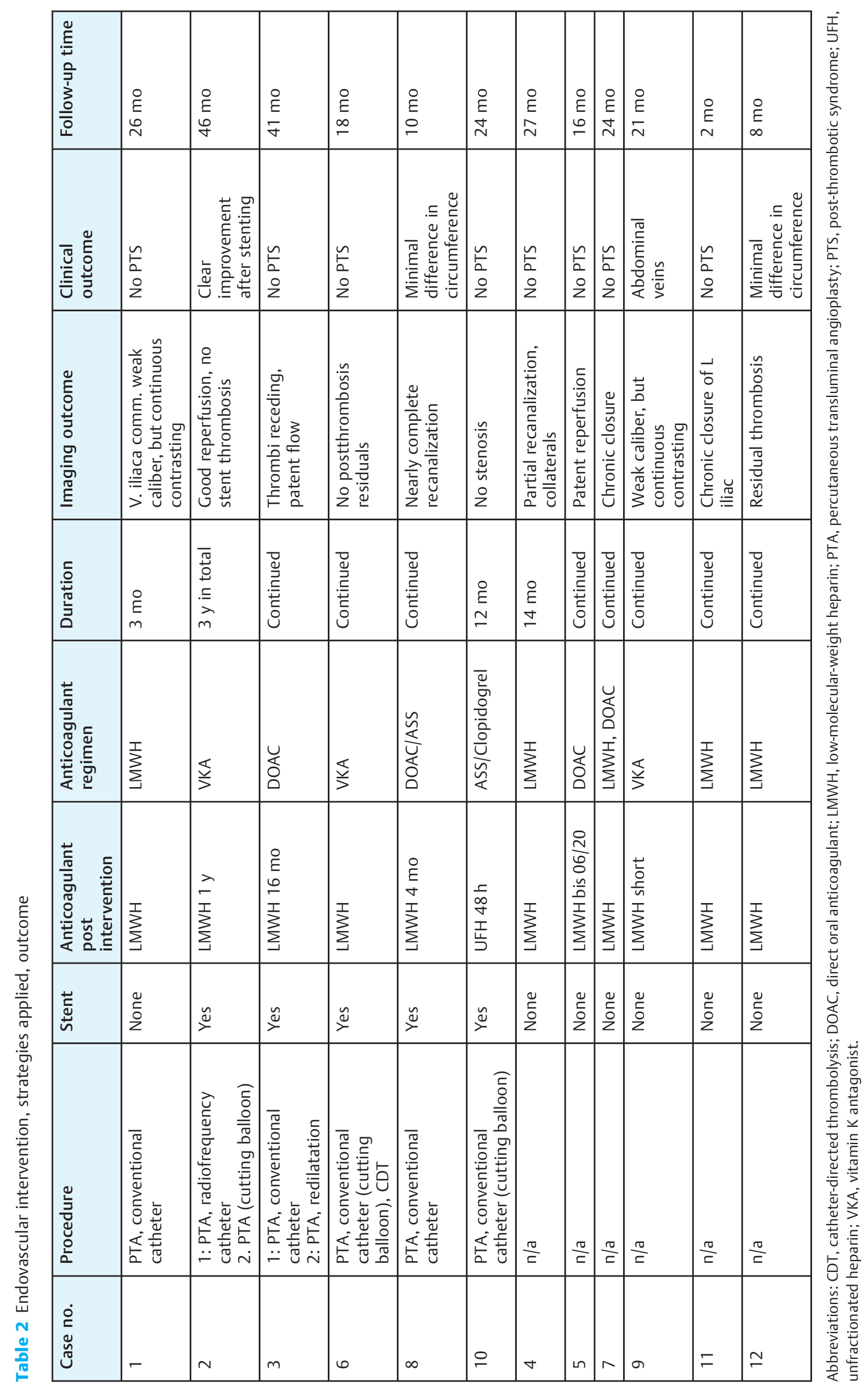


A
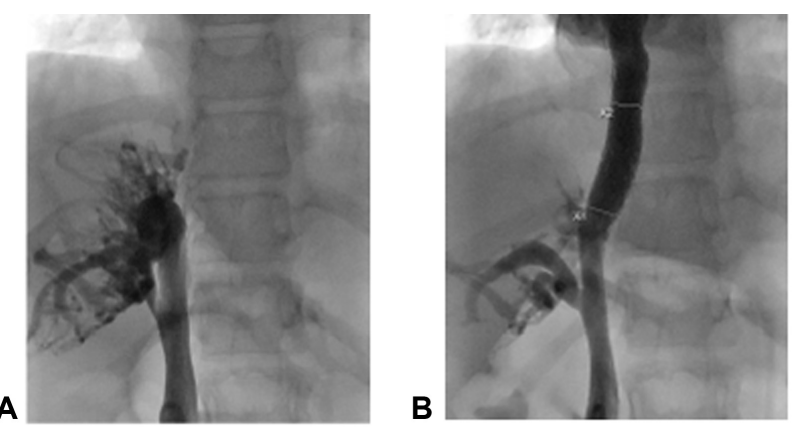

Fig. 1 Patient 10; inferior vena cava thrombosis before (A) and after percutaneous transluminal angioplasty (B).

anticoagulation, we analyzed the amount of thrombophilic risk factors (antiphospholipid antibodies, antinuclear antibodies, protein $\mathrm{C}$ deficiency, protein $\mathrm{S}$ deficiency, hetero/homozygous factor V-Leiden mutation, and factor II mutation) as well as positive family history and other risk factors for thrombosis (hormonal contraceptives, nicotine abuse, obesity, infection, immobilization). The average thrombophilic risk factors of patients receiving intervention was 1.16 versus 1 within the group not receiving intervention ( - Table 3 ). Due to its mild thrombophilic effect, we also calculated the differences without heterozygous factor $\mathrm{V}$ Leiden mutation (average: 0.71 vs. 0.5 ). The other risk factors like hormonal contraceptives, etc., did not differ substantially as well (average: 1.66 vs. 1). Due to low patient number, significance levels cannot be given, but the difference between predisposing risk factors in our cohort did not seem to influence the need for intervention.

\section{Discussion}

As the number of children with chronic diseases increases and because the rate of postthrombotic syndrome in adults can reach as high as $60 \%$, treatment options beyond anticoagulation in case of persistent VTE may need to be applied also in children. ${ }^{12,20}$ Postthrombotic syndrome is not only a
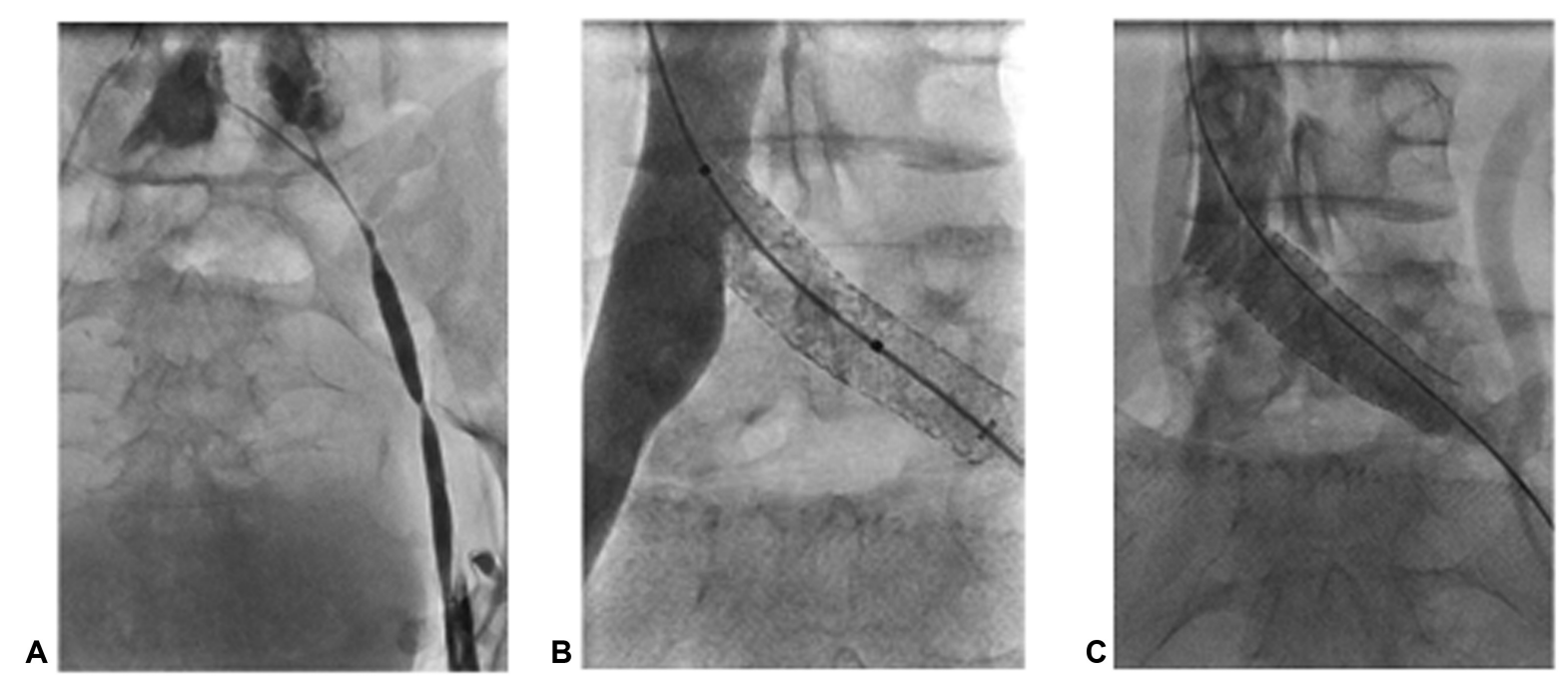

Fig. 2 Patient 2; thrombosis of left iliac and femoral vein before (A); while recanalization, percutaneous transluminal angioplasty, and stenting (B); and after stenting (C).

Table 3 Thrombophilia in patients with intervention and without intervention

\begin{tabular}{|c|c|c|c|c|c|c|c|c|c|}
\hline Intervention & $\begin{array}{l}\text { Pat. } \\
\text { no. }\end{array}$ & Thrombophilia & $\begin{array}{l}\text { Risk } \\
\text { factors }^{a}\end{array}$ & $\begin{array}{l}\text { Family } \\
\text { history }\end{array}$ & Intervention & $\begin{array}{l}\text { Pat. } \\
\text { no. }\end{array}$ & Thrombophilia & $\begin{array}{l}\text { Risk } \\
\text { factors }^{a}\end{array}$ & $\begin{array}{l}\text { Family } \\
\text { history }\end{array}$ \\
\hline \multirow[t]{6}{*}{ Yes } & 1 & FV het & 4 & 1 & \multirow[t]{6}{*}{ No } & 4 & - & 1 & 1 \\
\hline & 2 & FV het & 1 & + & & 5 & FV het & 1 & + \\
\hline & 3 & $\begin{array}{l}\text { FV het, } \\
\text { hypodys- } \\
\text { fibrinogenemia }\end{array}$ & 2 & + & & 7 & $\begin{array}{l}\text { FV het, } \\
\text { protein } C \text { het }\end{array}$ & 2 & + \\
\hline & 6 & Fll het, ANA & 1 & 1 & & 9 & AT III & 1 & 1 \\
\hline & 8 & prot-S het & 2 & - & & 11 & APLAs & 1 & 1 \\
\hline & 10 & - & 1 & 1 & & 12 & FV het & 2 & - \\
\hline & & Average: 1,16 & $\begin{array}{l}\text { Average: } \\
1,66\end{array}$ & & & & Average: 1 & Average: 1 & \\
\hline
\end{tabular}

Abbreviations: ANA, antinuclear antibodies; APLAs, antiphospholipid antibodies.

${ }^{a}$ Hormonal contraceptives, nicotine abuse, obesity, infection, immobilization.

|: No data available. 
cosmetic or comfort problem, but it imposes a high risk of reduced quality of life. ${ }^{21}$

In recent years, few publications showed that thrombolysis can be safely administered in children with total venoocclusive thrombosis to lower rates of postthrombotic syndrome. ${ }^{12,13,22-25}$ If residual thrombus mass exists, angioplasty and stenting can be used to reopen stenosis of veins.

Additionally, some studies used placement of an IVC filter (retrievable and nonretrievable). However, there are reports of accumulation of organized thrombus at the site of the IVC filter in nonretrievable IVC filters. ${ }^{13}$ Therefore, in 2020 a review by Kelkar et al emphasized on the importance of an evidence-based approach to IVC filters in adults, focusing its use mainly on patients with contraindication for anticoagulation. ${ }^{26}$

In 2018, the American Society of Hematology (ASH) published a guideline for the management of pediatric thrombosis. The authors emphasized that there is a wide variety of approaches on pediatric thrombosis and use of endovascular procedures, depending on local expertise. Therefore, the ASH recommendations 3 and 6 generally suggest against thrombolysis and thrombectomy followed by anticoagulation and support anticoagulation alone; however, they add the remark that in some patients thrombolysis or thrombectomy might be appropriate. ${ }^{27}$ Other guidelines suggest endovascular procedures in children with DVT only in life- or limb-threatening cases, or recommend thrombolytic options in cases in which "the benefits may outweigh the risks." ${ }^{10,28}$ Altogether, those recommendations stress the point that in certain cases, treatment options beyond anticoagulation are needed.

Bleeding is the most serious risk in patients undergoing endovascular thrombolysis. Major bleeding occurs in around $5.7 \%$ of children. ${ }^{27}$ In our case series, peri- or postprocedural complications were rare (one case of bleeding from puncture site). No major bleeding occurred.

A small meta-analysis of endovascular intervention in pediatric DVT reported similar safety rates in children, with two pulmonary embolisms among a total of 215 patients and two major hemorrhages. ${ }^{14}$

Locally recurrent DVT did not appear in our cohort, and redilatation was necessary in only two cases. All patients reported markedly improved clinical phenotype postprocedure.

The low rate of recurrence might be explained by the fact that the patients did not show any signs of a hypercoagulability state during the time of the procedure. Other studies reported recurrence rates of 27 and $12 \%{ }^{13,23}$ It can be hypothesized that late intervention leads to lower rates of recurrence as endothelial activation has reduced during the time from onset of thrombosis to intervention under anticoagulation therapy.

The low rate of postthrombotic syndrome in our intervention cohort (i.e., one patient with minimal difference in circumference) might be explained by the small cohort number, but it is in line with other findings of postthrombotic syndrome in $13 \%$ of patients at 1 and 2 years of followup $^{13}$ and $14 \%$ in the cohort described by Dandoy et al. ${ }^{22}$
There have been adult trials trying to improve outcome of percutaneous mechanical thrombolysis by adding systemic thrombolysis. These data showed that the rate of complete patency is significantly increased, and risk of postthrombotic syndrome is decreased. However, the rate of major bleeding also increased (nonsignificant). ${ }^{29}$ Thus, Enden et al suggested that additional catheter-directed thrombolysis should be applied in patients with proximal DVT and low risk of bleeding. ${ }^{30}$ So far, studies questioning systemic thrombolysis in combination with percutaneous mechanical thrombolysis have not been published in children, as risk of bleeding might be significantly increased.

This case series adds to the current knowledge, as it shows that even if not immediately applied, percutaneous mechanical thrombolysis can improve outcome regarding postthrombotic syndrome. Our small case series shows that even in chronic VTE, interventional procedures can improve outcome.

The patients of this cohort, not receiving intervention, showed either partial or complete reperfusion or had developed patent collateralization and none of the patients developed postthrombotic syndrome. Therefore, anticoagulation alone is still a treatment option if clinical symptoms do clearly improve within the first months of anticoagulant treatment.

Limitations to this study are its retrospective design and small sample size. This might cause underreporting of postthrombotic syndrome. However, the mean follow-up time of 26 months is comparably high and all patients are still regularly visiting our department and are screened for clinical symptoms.

In summary, our data show that in case of secondary endovascular intervention, applied in pediatric patients with persistent clinical signs of venous occlusion, improvement can be achieved, even if intervention is performed months after diagnosis and initial anticoagulation treatment.

\section{Conflict of Interest}

B.Z. reports grants from Takeda, CSL Behring, Biotest to the University Medical Center Freiburg.

B.S. reports grants from Studie CV185-362, Apixaban for Bristol-Myers Squibb GmbH \& Co. KGaA Arnulfstraße 29, and D-80636 München.

B.S. reports participation on a Data Safety Monitoring Board or Advisory Board from Studie CV185-362, Apixaban for Bristol-Myers Squibb GmbH \& Co. KGaA Arnulfstraße 29, and D-80636 München.

\section{References}

1 Andrew M, David M, Adams M, et al. Venous thromboembolic complications (VTE) in children: first analyses of the Canadian Registry of VTE. Blood 1994;83(05):1251-1257

2 van Ommen CH, Heijboer H, Büller HR, Hirasing RA, Heijmans HS, Peters M. Venous thromboembolism in childhood: a prospective two-year registry in the Netherlands. J Pediatr 2001;139(05): 676-681 
3 Raffini L, Huang Y-S, Witmer C, Feudtner C. Dramatic increase in venous thromboembolism in children's hospitals in the United States from 2001 to 2007. Pediatrics 2009;124(04):1001-1008

4 Stubbs JM, Assareh H, Curnow J, Hitos K, Achat HM. Incidence of in-hospital and post-discharge diagnosed hospital-associated venous thromboembolism using linked administrative data. Intern Med J 2018;48(02):157-165

5 Zhang Z, Lei J, Shao X, et al; China Venous Thromboembolism Study Group. Trends in hospitalization and in-hospital mortality from VTE, 2007 to 2016, in China. Chest 2019;155(02):342-353

6 Mahajerin A, Betensky M, Goldenberg NA. Thrombosis in children: approach to anatomic risks, thrombophilia, prevention, and treatment. Hematol Oncol Clin North Am 2019;33(03):439-453

7 Lassandro G, Palmieri VV, Palladino V, Amoruso A, Faienza MF, Giordano P. Venous thromboembolism in children: from diagnosis to management. Int J Environ Res Public Health 2020;17(14):

8 Spentzouris G, Scriven RJ, Lee TK, Labropoulos N. Pediatric venous thromboembolism in relation to adults. J Vasc Surg 2012;55(06): 1785-1793

9 Massicotte P, Adams M, Marzinotto V, Brooker LA, Andrew M. Low-molecular-weight heparin in pediatric patients with thrombotic disease: a dose finding study. J Pediatr 1996;128(03): 313-318

10 Monagle P, Chan AKC, Goldenberg NA, et al. Antithrombotic therapy in neonates and children: antithrombotic therapy and prevention of thrombosis, 9th ed: American College of Chest Physicians Evidence-Based Clinical Practice Guidelines. Chest 2012;141(2, Suppl):e737S-e801S

11 Monagle P, Chalmers E, Chan A, et al. Antithrombotic therapy in neonates and children: American College of Chest Physicians Evidence-Based Clinical Practice Guidelines (8th edition). Chest 2008;133(6, Suppl):887S-968S

12 Goldenberg NA, Donadini MP, Kahn SR, et al. Post-thrombotic syndrome in children: a systematic review of frequency of occurrence, validity of outcome measures, and prognostic factors. Haematologica 2010;95(11):1952-1959

13 Goldenberg NA, Branchford B, Wang M, Ray C Jr, Durham JD, Manco-Johnson MJ. Percutaneous mechanical and pharmacomechanical thrombolysis for occlusive deep vein thrombosis of the proximal limb in adolescent subjects: findings from an institution-based prospective inception cohort study of pediatric venous thromboembolism. J Vasc Interv Radiol 2011;22(02): 121-132

14 Tarango C, Manco-Johnson MJ. Pediatric thrombolysis: a practical approach. Front Pediatr 2017;5:260-260

15 Kearon C, Akl EA, Comerota AJ, et al. Antithrombotic therapy for VTE disease: antithrombotic therapy and prevention of thrombosis, 9th ed: American College of Chest Physicians Evidence-Based Clinical Practice Guidelines. Chest 2012;141(2, Suppl): e419S-e496s

16 Strijkers RHW, de Wolf MAF, Arnoldussen CWKP, et al. Venous instent thrombosis treated by ultrasound accelerated catheter directed thrombolysis. Eur J Vasc Endovasc Surg 2015;49(04): 440-447

17 Zaghlool DS, Franz RW, Jenkins J. EkoSonic thrombolysis as a therapeutic adjunct in venous occlusive disease. Int J Angiol 2016; 25(04):203-209

18 Albisetti M. Thrombolytic therapy in children. Thromb Res 2006; 118(01):95-105

19 Yee DL, Chan AKC, Williams S, Goldenberg NA, Massicotte MP, Raffini LJ. Varied opinions on thrombolysis for venous thromboembolism in infants and children: findings from a survey of pediatric hematology-oncology specialists. Pediatr Blood Cancer 2009;53(06):960-966

20 Kuhle S, Koloshuk B, Marzinotto V, et al. A cross-sectional study evaluating post-thrombotic syndrome in children. Thromb Res 2003;111(4-5):227-233

21 Manco-Johnson MJ. Postthrombotic syndrome in children. Acta Haematol 2006;115(3-4):207-213

22 Dandoy CE, Kukreja KU, Gruppo RA, Patel MN, Tarango C. Outcomes in children with deep vein thrombosis managed with percutaneous endovascular thrombolysis. Pediatr Radiol 2015; 45(05):719-726

23 Gaballah M, Shi J, Kukreja K, et al. Endovascular thrombolysis in the management of iliofemoral thrombosis in children: a multiinstitutional experience. J Vasc Interv Radiol JVIR 2016;27(04): 524-530

24 Goldenberg NA, Durham JD, Knapp-Clevenger R, Manco-Johnson MJ. A thrombolytic regimen for high-risk deep venous thrombosis may substantially reduce the risk of postthrombotic syndrome in children. Blood 2007;110(01):45-53

25 Darbari DS, Desai D, Arnaldez F, et al. Safety and efficacy of catheter directed thrombolysis in children with deep venous thrombosis. Br J Haematol 2012;159(03):376-378

26 Kelkar AH, Rajasekhar A. Inferior vena cava filters: a framework for evidence-based use. Hematol Am Soc Hematol Educ Program 2020;2020(01):619-628

27 Monagle P, Cuello CA, Augustine C, et al. American Society of Hematology 2018 Guidelines for management of venous thromboembolism: treatment of pediatric venous thromboembolism. Blood Adv 2018;2(22):3292-316

28 Jaff MR, McMurtry MS, Archer SL, et al. Management of massive and submassive pulmonary embolism, iliofemoral deep vein thrombosis, and chronic thromboembolic pulmonary hypertension: a scientific statement from the American Heart Association. Circulation 2011;123(16):1788-830

29 Watson L, Broderick C, Armon MP. Thrombolysis for acute deep vein thrombosis. Cochrane Database Syst Rev 2016;11(11): CD002783

30 Enden T, Kløw N-E, Sandvik L, et al. Catheter-directed thrombolysis vs. anticoagulant therapy alone in deep vein thrombosis: results of an open randomized, controlled trial reporting on short-term patency. J Thromb Haemost JTH 2009;7(08): 1268-1275 